\title{
Full Ionisation in Binary-Binary Scattering
}

\author{
W. L. Sweatman \\ Institute of Information and Mathematical Sciences, Massey University at Albany, Private Bag \\ 102 904, North Shore 0745, New Zealand \\ email: w.sweatman@massey.ac.nz
}

\begin{abstract}
Encounters between binary stars and single stars and between binary stars and other binary stars play a key role in the dynamics of dense stellar systems. In the simple model, in which stars are approximated by point masses, a number of theoretical and numerical results are known. In particular there exist relationships to help to describe the destruction process of binary stars (ionisation) through three-body encounters between binary and single stars. Here we extend these results to the four-body case involving encounters between pairs of binary stars that lead to a disruption of the binaries into single stars.
\end{abstract}

Keywords. binaries: general, scattering, stellar dynamics, celestial mechanics

\section{Introduction}

In dense stellar systems, interactions involving binaries are important for energy transfer. In addition, binaries can be created and destroyed. The most likely encounters are those between a single star and a binary star and those between a pair of binary stars. In the simplest model, which is used here, the stars are approximated by point masses. Early studies incorporating simulations of binary-binary interactions extend back to those of Mikkola 1983 and Hoffer 1983. Numerical simulations that support the theory developed in the present investigation made use of the CHAIN code developed by Mikkola \& Aarseth (Mikkola \& Aarseth 1993, 1996; Aarseth 2003).

\section{Full Ionisation}

In full ionisation all binaries involved in a scattering encounter are destroyed to create a number of separating single masses. The likelihood is measured by the total crosssection $(\sigma)$. A key parameter is the total energy $(h)$ in the centre of mass rest frame or the closely related relative velocity of the two approaching objects at infinity $\left(v_{\infty}\right)$. Full ionisation is impossible for negative total energy. As total energy increases from zero, the cross-section rapidly rises from zero, peaks, and then steadily declines. This evolution has been illustrated and described in the four-body binary-binary case (Hut 1992; Sweatman 2007) as well as for three bodies (Hut \& Bahcall 1983; Hut 1983).

Considering binary-binary interactions with equal masses $(m)$, here we summarise the low energy results of Sweatman (2007) and then present new results for high energy.

\subsection{Full ionisation in binary-binary encounters at low positive total energy}

Here, full ionisation is barely possible. The energy needs to be distributed quite precisely among the masses, with comparable inter-body distances for a period of expansion. The orbit must pass close to a central configuration (Heggie \& Sweatman 1991; Heggie \& Hut 1993, 2003) and the cross-section is found to be proportional to $h^{\beta}$ where $\beta=$ $\sum_{\operatorname{Re} \lambda_{i}>0, \lambda_{i} \neq 2 / 3} 3 \operatorname{Re} \lambda_{i} / 2$, and the $\lambda_{i}$ are related to the 'Siegel exponents' (Siegel \& 
Moser 1971). With sufficiently small energy, the dominant configuration gives

$$
\sigma \propto h^{\beta} \text { with } \beta=-\frac{1}{2}+\frac{1}{2} \sqrt{23-4 \sqrt{3}+2 \sqrt{469-236 \sqrt{3}}} \approx 2.310 .
$$

\subsection{Full ionisation in binary-binary encounters at high total energy}

Here, ionisation cross-section decreases due to the requirement for sufficient momentum transfer during the brief close encounter. The asymptotic relationship $\sigma \propto 1 / v_{\infty}^{2}$ derived in the three-body case (Heggie 1975; Hut 1983), has also been found in the four-body case (Hut 1992; Sweatman 2007). In the present investigation, an approach, similar to that of Hut (1983), has been used to find the coefficient of proportionality in various four-body cases. More details will be included in a forthcoming publication. The results summarised below apply to two circular binaries with semi-major axes $a$ and $a Q$, where $Q$, the ratio of the axes, is taken to be greater than or equal to 1 .

When $Q$ is between 1 and $17+12 \sqrt{2} \approx 33.97$ the full ionisation cross-section is

$$
\sigma=\left(\frac{40}{3}+\frac{\left(1+2 \beta^{2}\right) Q^{\frac{1}{2}}+2+\beta^{2}}{12 \beta^{4} Q^{\frac{1}{2}}}\left(Q^{\frac{1}{2}}-\beta^{2}\right)^{3}+\frac{Q-\beta^{4}}{2 \beta^{2}}+\frac{Q^{\frac{1}{2}}}{2} \ln \left[\frac{\beta^{4}}{Q}\right]\right) \frac{\pi a G m}{v_{\infty}^{2}}
$$

where $\beta=\sqrt{2}+1$. So that if the binaries have equal binding energies $(Q=1)$, then

$$
\sigma=\left(\frac{40}{3}-6 \sqrt{2}+2 \ln (\sqrt{2}+1)\right) \frac{\pi a G m}{v_{\infty}^{2}}
$$

approximately 0.9916 of the three-body ionisation cross-section with the same $a$ and $v_{\infty}$.

If $Q$ is greater than $17+12 \sqrt{2}$ then all interactions ionising the tight binary will also ionise the loose binary: in any orientation the maximum ionisation impact parameter of the tightly bound binary is less than that of the other binary. In this case the four-body full ionisation cross-section is exactly twice that for an interaction between a single star and the tight binary, the factor of two being due to the two stars in the loose binary:

$$
\sigma=\frac{40}{3} \frac{\pi a G m}{v_{\infty}^{2}}
$$

\section{References}

Aarseth, S. J. 2003, Gravitational N-Body Simulations, (Cambridge: CUP)

Heggie, D. C. 1975, MNRAS 173, 729

Heggie, D. C. \& Hut, P. 1993, ApJS 85, 347

Heggie, D. C. \& Hut, P. 2003, The Gravitational Million-Body Problem, (Cambridge: CUP)

Heggie, D. C. \& Sweatman, W. L. 1991, MNRAS 250, 555

Hoffer, J. B. 1983, $A J$ 88, 1420

Hut, P. 1983, ApJ 268, 342

Hut, P. 1992, in: E. P. J. van der Heuvel \& S. A. Rappaport (eds.), X-ray Binaries and Recycled Pulsars, (Dordrecht: Kluwer), p. 317

Hut, P. \& Bahcall, J. N. 1983, ApJ 268, 319

Mikkola, S. 1983, MNRAS 203, 1107

Mikkola, S. \& Aarseth, S. J. 1993, Cel. Mech. 57, 439

Mikkola, S. \& Aarseth, S. J. 1996, Cel. Mech. 64, 197

Siegel, C. L. \& Moser, J. K. 1971, Lectures on Celestial Mechanics, (Berlin: Springer-Verlag)

Sweatman, W. L. 2007, MNRAS 377, 459 